\title{
Molecular tumor testing in patients with Lynch-like syndrome reveals a de novo mosaic variant of a mismatch repair gene transmitted to offspring
}

\author{
Erell Guillerm ${ }^{1,2,3} \cdot$ Magali Svrcek $^{2,3,4} \cdot$ Armelle Bardier-Dupas $^{5} \cdot$ Noémie Basset $^{1,2,3} \cdot$ Florence Coulet $^{1,2,3}$. \\ Chrystelle Colas ${ }^{1,2,3,6}$
}

Received: 18 February 2018 / Revised: 19 February 2020 / Accepted: 18 June 2020 / Published online: 16 July 2020

(c) European Society of Human Genetics 2020

\begin{abstract}
In Lynch-like syndrome, patients have tumors with microsatellite instability but no germline pathogenic variant in mismatch repair genes or somatic methylation of the $M L H 1$ promoter. Identification of the mechanism that causes these tumors is crucial for guiding screening of the patients and their relatives. Double somatic hits are the usual explanation for these cases; however, we have previously reported a de novo mosaic pathogenic variant in a patient with Lynch-like syndrome. Using tumoral NGS analysis of a series of 16 patients with Lynch-like syndrome, we found six patients with double somatic hits, including one patient with mosaicism of a de novo pathogenic variant in MSH2. This variant was transmitted to the patient's offspring, which has significant implications for genetic counseling.
\end{abstract}

\section{Introduction}

Lynch syndrome (LS) is a predisposition to colorectal and endometrial cancer due to a germline pathogenic variant in one of the mismatch repair (MMR) genes. The MMR defect results in tumors with microsatellite instability (MSI) and/or loss of expression of one of the MMR proteins, as detected by immunochemistry (IHC). MSI and loss of MLH1 expression can also be due to hypermethylation of the $M L H 1$ promoter in

Erell Guillerm

erell.guillerm@aphp.fr

1 Department of Genetics, Hôpital Universitaire Pitié Salpêtrière (Assistance Publique-Hôpitaux de Paris), Paris VI University, Paris, France

2 INSERM, UMRS 938-Centre de Recherche Saint-Antoine, Equipe 'Instabilité des Microsatellites et Cancers', Equipe labellisée par la Ligue Nationale contre le Cancer, Paris, France

3 France Université Pierre et Marie Curie, Paris, France

4 Department of Pathology, Hôpital Saint Antoine (Assistance Publique-Hôpitaux de Paris), Paris VI University, Paris, France

5 Department of Pathology, Hôpital Universitaire Pitié Salpêtrière (Assistance Publique-Hôpitaux de Paris), Paris VI University, Paris, France

6 Present address: Department of Genetics, Curie Institute, Paris, France sporadic colorectal cancer (CRC) cases. In cases where germline analysis fails to identify a pathogenic variant, the patient is considered to have Lynch-like syndrome (LLS) [1-3]. In this situation, it is recommended that all family members undergo regular screening, as their cancer risk is known to be higher than that of the general population $[4,5]$. Double somatic pathogenic variants have been described as the most frequent mechanism underlying some of these cases [6-9]. These patients are sporadic cases with, theoretically, no risk of transmission. Here, we report a series of LLS patients, including one case of mosaicism of a de novo pathogenic variant of $M S H 2$ that was transmitted to offspring.

\section{Material and methods}

From January 2005 to December 2016, 16 LLS patients (included one previously reported [9]) for whom frozen tumoral tissue was available were selected. All patients gave informed consent in accordance with French law. All had CRC with MSI and loss of expression of at least one MMR protein (Table 1). All had previously tested negative for germline pathogenic variants in $M L H 1, M S H 2, E P C A M$, MSH6, and PMS2, by NGS analysis and MLPA, using methods described in detail below. In the six patients with loss of MLH1 expression, no $M L H 1$ promoter methylation was detected. In the ten patients with loss of MSH2 


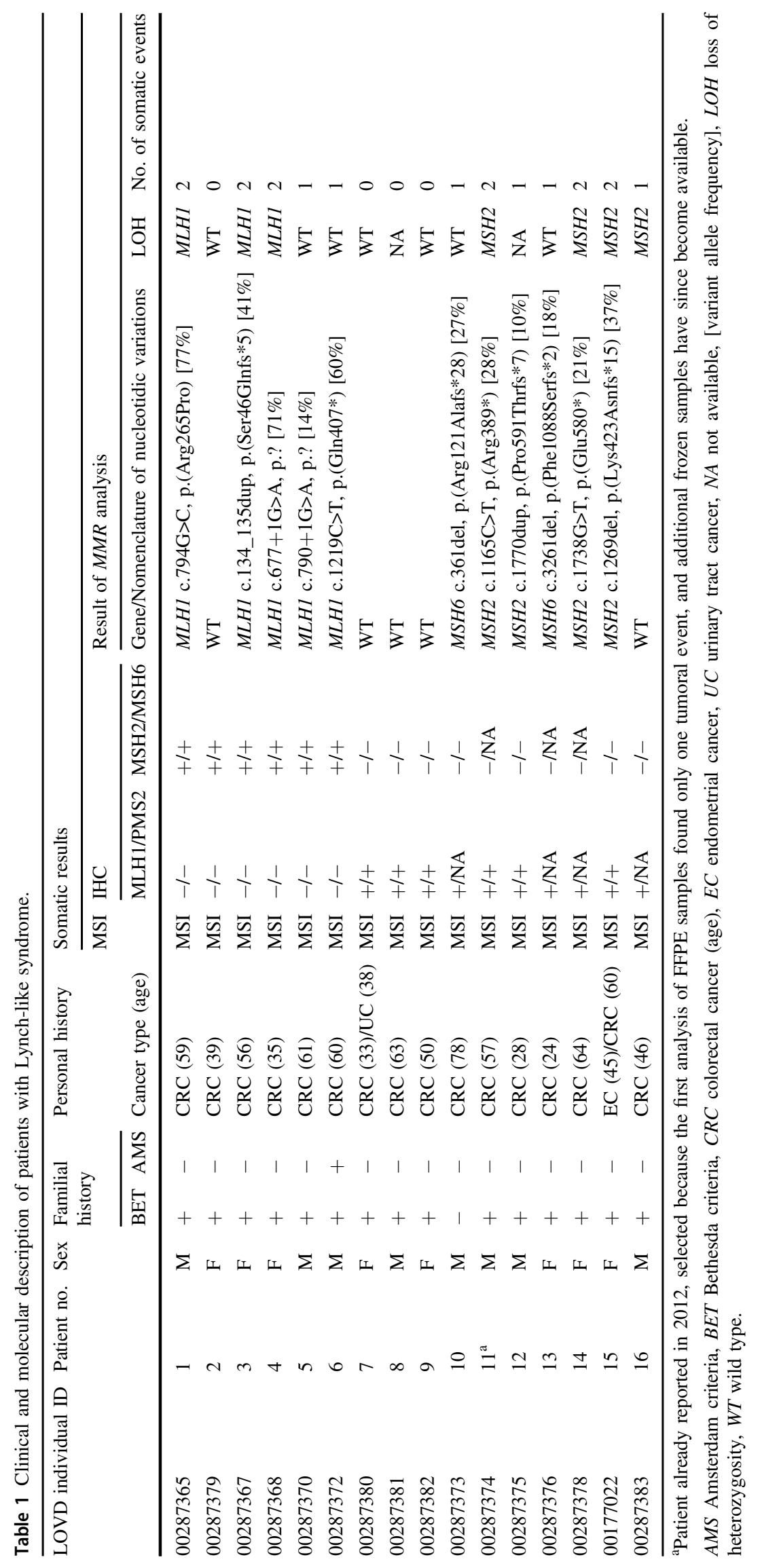


A

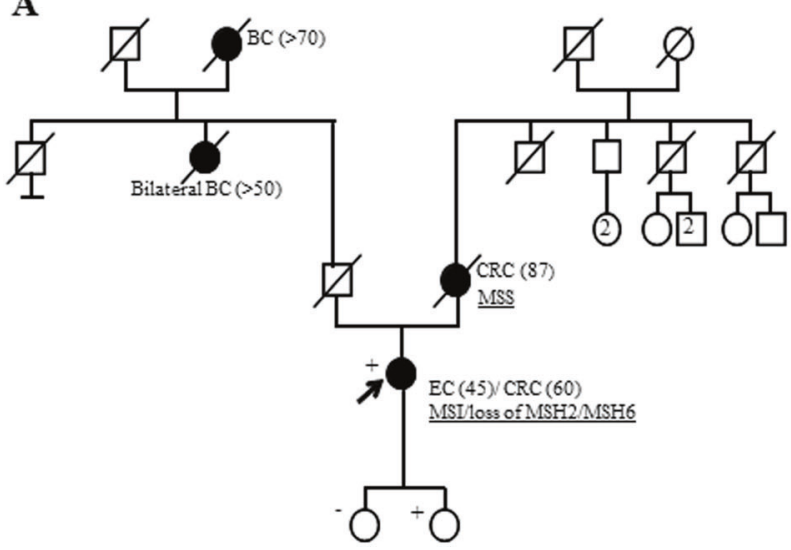

Fig. 1 Description of patient 15, who has mosaicism of the de novo MSH2 pathogenic variant. a Pedigree of patient 15 . b Sanger sequencing results of the $M S H 2$ gene for patient 15 (different samples) and her daughter. BC breast cancer, CRC colorectal cancer, EC

expression, paracentric inversion of chromosome 2 was not found by either karyotype analysis or MLPA [10].

We analyzed the four $M M R$ genes in frozen tumor tissue samples by NGS using the HNPCC MASTR kit/Plus kit (Multiplicom, Belgium). Libraries were sequenced using the Illumina MiSeq Sequencing system. The data were processed to obtain minimal coverage at $\times 200$. The resulting sequence data were analyzed using SeqNext software (adapted from BWA and Smith-Waterman algorithms with a cutoff at $15 \%$ ), version 4.2.2 (JSI Medical Systems, Germany), and compared with the reference sequences for MLH1, MSH2, MSH6, and PMS2 (NM_000249.3, NM_000251.2, NM_000179.2, and NM_000535.5, respectively). Screening for large rearrangements or loss of heterozygosity was performed by MLPA using the SALSAP003 kit for MLH1/MSH2/EPCAM and the SALSAP072 kit for MSH6 (MRC Holland, Holland). This analysis was carried out using the ABI 3730 DNA analyzer (Applied Biosystems, USA) and GeneMapper software, version 5 (Applied Biosystems, USA).

When a tumoral pathogenic variant was found, we performed a targeted re-examination of the germline NGS results with lower detection rates and targeted Sanger analysis in normal adjacent tissue, and in lymphocytes from the patient's offspring, when available. The Sanger sequencing data were analyzed using SeqScape software, version 2.6 (Thermo Fisher) (Mixed bases settings $>15 \%$ ). The data were deposited in LOVD database at https://insight-database.org (Table 1).

\section{Results}

Molecular analysis of $M M R$ genes in tumor samples from the 16 patients found six biallelic somatic events. The second event was always loss of heterozygosity, consistent with the literature [11]. Six tumors had only one somatic
B

Proband's blood

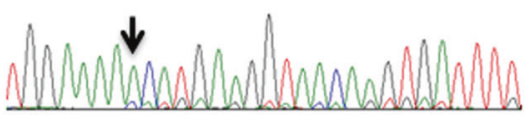

Proband's normal colon

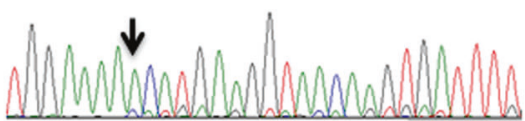

Proband's CRC

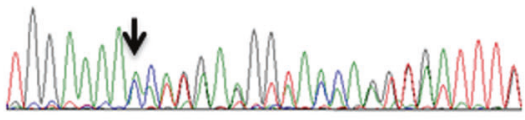

Daughter's blood

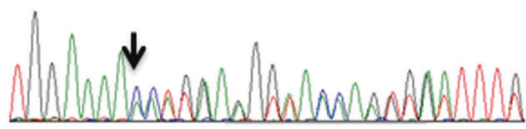

endometrial cancer, (age at diagnosis), underlined: somatic results, - : noncarrier of the pathogenic variant, + : carrier of the pathogenic variant. The arrow indicates the position of the variant c.1269del, p. (Lys423Asnfs*15).

event (Table 1). We searched all pathogenic variants in normal adjacent tissue for 7 out of the 12 patients who had at least one tumoral genetic event, and in lymphocytes from the offspring of patient 15 . We identified one case of mosaic LLS with transmission to offspring (Fig. 1). Patient 15 developed endometrial cancer at age 45 and colon cancer at age 60 . The colon cancer showed MSI, and both tumors had loss of MSH2 and MSH6 expression. Molecular analysis of the colon cancer tissue identified a variant of $\mathrm{MSH} 2$ that is predicted to affect protein function: c.1269del, p. (Lys423Asnfs*15). Re-examination of the NGS data revealed that this variant appeared at a $9 \%$ allele frequency, which is below our usual detection limit. In normal colon tissue, targeted Sanger sequencing showed a weak signal corresponding to the frameshift variant that was slightly higher than the background noise. One of the two patient's daughters is heterozygous for this variant (Fig. 1). The patient's mother developed CRC at age 87 . Her tumor was microsatellite stable with normal IHC, which is consistent with a phenocopy, supporting the diagnosis of a de novo somatic and germline mosaic variant for patient 15 .

\section{Discussion}

Out of six patients with double somatic hits, we found one with mosaicism of a de novo pathogenic variant. We only detected one event in ten patients' tumors. Further investigations such as analysis of other genes that, when mutated, mimic LS (e.g., POL, MUTYH) and RNA studies are currently underway for these patients $[8,12-16]$.

Pastrello et al. were the first to show that LS could arise from mutations that are undetectable in blood DNA due to reversion of an inherited mutation [17]. In 2013, we described the first case of germline mosaicism in $\mathrm{MSH} 2$ in 
Fig. 2 Decision tree for exploring suspected mosaicism in patients with Lynch-like syndrome. PST presymptomatic testing.

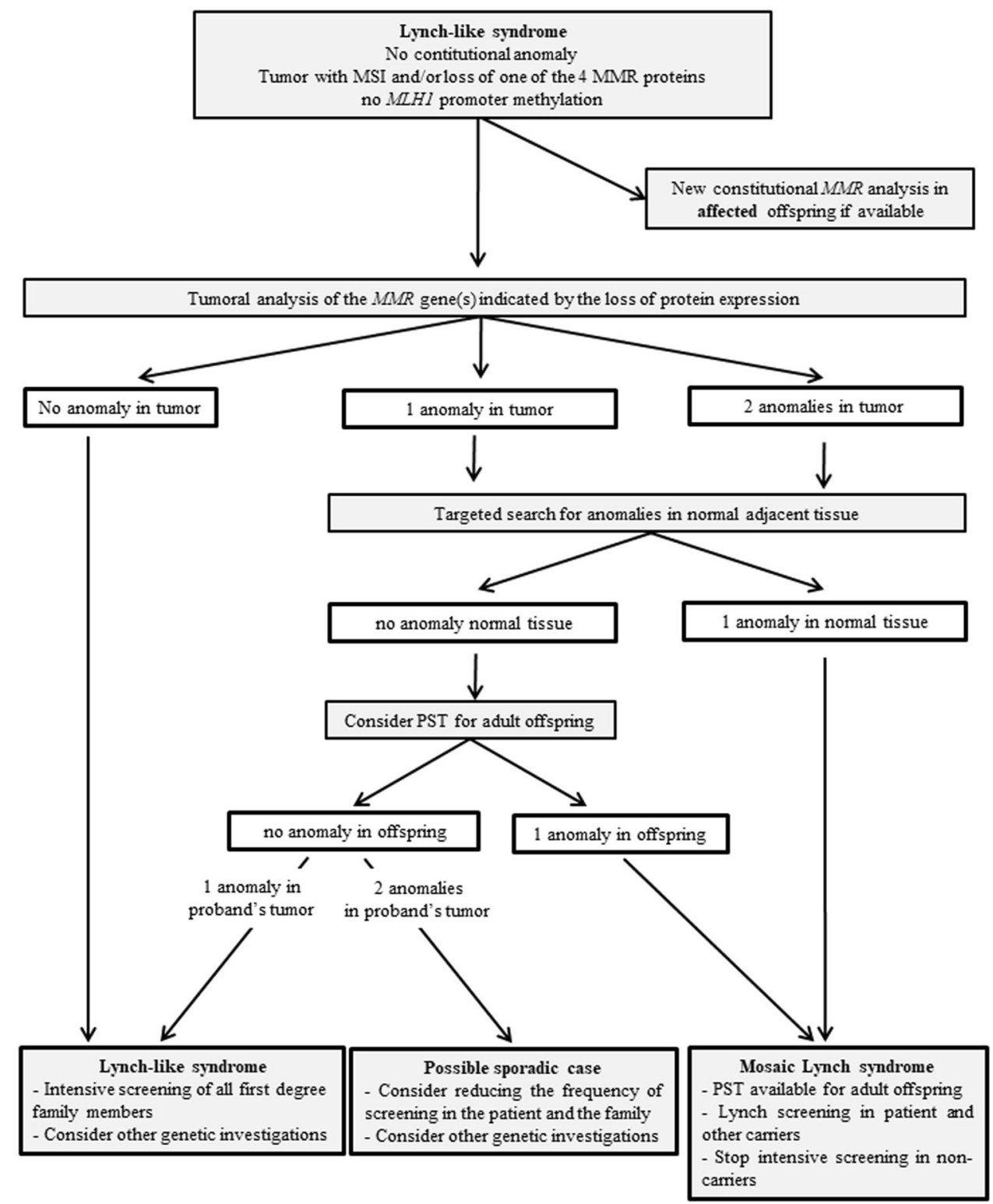

one out of five patients with double somatic hits [9]. This $\mathrm{MSH} 2$ mutation was found in a patient with LS and in CRC tissue from his mother, but was not observed in the mother's normal colon tissue or lymphocytes [9]. The transmission to her son indicated germline mosaicism, but the level of mosaicism was too low to be detected in normal tissues, due to technical limitations of Sanger sequencing. Deep NGS sequencing revealed that $1.7 \%$ of reads $(7000 \times)$ from- her lymphocytes contained the mutation.

Regardless of the disease, the frequency of mosaicism is correlated with the frequency of de novo events. De novo mutations in LS have already been described, and while their frequency is low $(2.3 \%)$, it is probably underestimated [18]. De novo variants occurring during embryonic development may lead to mosaic cases. Parental somatic mosaicism is known to be the cause of other cancer predispositions
$[19,20]$. We do not expect to observe a family history in the siblings or parents of the person in whom mosaicism occurs. However, some common cancers may be reported in the family and proved to be phenocopies (as in mother of patient 15). A familial history of Lynch related tumors should not rule out the assumption of mosaicism.

In both families that we have reported, the patients with mosaicism had CRC. For patient 15, endometrial cancer also occurred; and transmission to offspring means that the variant was also present in the gonads. The involvement of these different tissues illustrates that the mutation occurred early in development, before the end of the third week of embryogenesis.

The most frequent explanation for LLS is double somatic hits. These patients are usually considered to be sporadic, and intensive screening of their family members is therefore not 
performed $[1,6,7,12]$. However, if mosaicism of a pathogenic variant is found in the patient, intensive screening should be continued for both the patient and the patient's offspring who carry this variant. Screening can be halted for children who do not carry this variant, as well as for siblings who are not at risk of having it. Identification of the germline pathogenic variant in the patient is essential for providing genetic testing and accurate risk estimation for relatives. In Fig. 2, we propose an algorithm to be used for research purposes for assessing LLS patients and their relatives in order to detect mosaicism and establish a suitable screening approach for the family. We recommend (1) starting germline screening of the youngest generation when two generations suffer from cancer, (2) analyzing the MMR genes in tumors from LLS patients, and (3) testing offspring when one or two pathogenic variants are found in the tumor, even if those variants are absent in normal adjacent tissue or in the proband's lymphocytes. NGS can fail to detect variants in lymphocytes or normal tissue, depending on the sequencing depth selected. Deep sequencing analysis may help detect variants with low allele frequency, but tumoral analysis remains essential to target the variant in the germline study.

The second mosaic case described in this study confirms that mosaicism could explain some cases of LLS. This might not be a rare mechanism, as it was found in 2/33 LLS patients. We are currently establishing collaborations to enlarge this study by including more cases and FFPE tissues, in order to determine the prevalence of this type of event and the cost-effectiveness of our algorithm.

Acknowledgements The authors wish to thank the Tumorothèque des Hôpitaux Universitaires de l'Est Parisien (HUEP), AP-HP, Hôpital Tenon, the Service d'Anatomie Pathologique, F-75970 Paris, France, and the Centre de Ressources Biologiques du service d'Anatomie et Cytologie Pathologiques de la Pitié-Salpêtrière, AP-HP, F-75013 Paris, France, for providing the frozen tumor samples.

Funding This work was supported by Assistance Publique-Hôpitaux de Paris and by grants from the Institut National du Cancer (INCa).

\section{Compliance with ethical standards}

Conflict of interest The authors declare that they have no conflict of interest.

Publisher's note Springer Nature remains neutral with regard to jurisdictional claims in published maps and institutional affiliations.

\section{References}

1. Carethers JM. Differentiating Lynch-like from Lynch syndrome. Gastroenterology. 2014;146:602-4.

2. Boland CR. The mystery of mismatch repair deficiency: Lynch or Lynch-like? Gastroenterology. 2013;144:868-70.
3. Stoffel EM, Boland CR. Genetics and genetic testing in hereditary colorectal cancer. Gastroenterology. 2015;149:1191-203.

4. Buchanan DD, Rosty C, Clendenning M, Spurdle AB, Win AK. Clinical problems of colorectal cancer and endometrial cancer cases with unknown cause of tumor mismatch repair deficiency (suspected Lynch syndrome). Appl Clin Genet. 2014;7:183-93.

5. Rodríguez-Soler M, Pérez-Carbonell L, Guarinos C, Zapater P, Castillejo A, Barberá VM, et al. Risk of cancer in cases of suspected lynch syndrome without germline mutation. Gastroenterology. 2013;144:926-32.

6. Haraldsdottir S, Hampel H, Tomsic J, Frankel WL, Pearlman R, de la Chapelle A, et al. Colon and endometrial cancers with mismatch repair deficiency can arise from somatic, rather than germline, mutations. Gastroenterology. 2014;147:1308-16.

7. Geurts-Giele WRR, Leenen CHM, Dubbink HJ, Meijssen IC, Post E, Sleddens HF, et al. Somatic aberrations of mismatch repair genes as a cause of microsatellite-unstable cancers. J Pathol. 2014;234:548-59.

8. Vargas-Parra GM, González-Acosta M, Thompson BA, Gómez C, Fernández A, Dámaso E, et al. Elucidating the molecular basis of MSH2-deficient tumors by combined germline and somatic analysis. Int J Cancer. 2017;141:1365-80.

9. Sourrouille I, Coulet F, Lefevre JH, Colas C, Eyries M, Svrcek M, et al. Somatic mosaicism and double somatic hits can lead to MSI colorectal tumors. Fam Cancer. 2013;12:27-33.

10. Wagner A, van der Klift H, Franken P, Wijnen J, Breukel C, Bezrookove $\mathrm{V}$, et al. A $10-\mathrm{Mb}$ paracentric inversion of chromosome arm $2 p$ inactivates MSH2 and is responsible for hereditary nonpolyposis colorectal cancer in a North-American kindred. Genes Chromosomes Cancer. 2002;35:49-57.

11. Carethers JM, Stoffel EM. Lynch syndrome and Lynch syndrome mimics: the growing complex landscape of hereditary colon cancer. World J Gastroenterol. 2015;21:9253-61.

12. Mensenkamp AR, Vogelaar IP, van Zelst-Stams WAG, Goossens M, Ouchene H, Hendriks-Cornelissen SJ, et al. Somatic mutations in MLH1 and MSH2 are a frequent cause of mismatch-repair deficiency in Lynch syndrome-like tumors. Gastroenterology. 2014;146:643-6.

13. Morak M, Heidenreich B, Keller G, Hampel H, Laner A, de la Chapelle A, et al. Biallelic MUTYH mutations can mimic Lynch syndrome. Eur J Hum Genet. 2014;22:1334-7.

14. Lefevre JH, Colas C, Coulet F, Bonilla C, Mourra N, Flejou JF, et al. MYH biallelic mutation can inactivate the two genetic pathways of colorectal cancer by APC or MLH1 transversions. Fam Cancer. 2010;9:589-94.

15. Castillejo A, Vargas G, Castillejo I, Navarro M, Barberá VM, González S, et al. Prevalence of germline MUTYH mutations among Lynch-like syndrome patients. Eur J Cancer. 2014;50:2241-50.

16. Elsayed F, Kets CM, Ruano D, van den Akker B, Mensenkamp AR, Schrumpf M, et al. Germline variants in POLE are associated with early onset mismatch repair deficient colorectal cancer. EJHG. 2015;23:1080-4.

17. Pastrello C, Fornasarig M, Pin E, Berto E, Pivetta B, Viel A. Somatic mosaicism in a patient with Lynch syndrome. Am J Med Genet A. 2009;149A:212-5.

18. Win AK, Jenkins MA, Buchanan DD, Clendenning M, Young JP, Giles GG, et al. Determining the frequency of de novo germline mutations in DNA mismatch repair genes. J Med Genet. 2011;48:530-4.

19. Spier I, Drichel D, Kerick M, Kirfel J, Horpaopan S, Laner A, et al. Low-level APC mutational mosaicism is the underlying cause in a substantial fraction of unexplained colorectal adenomatous polyposis cases. J Med Genet. 2016;53:172-9.

20. Dehainault C, Golmard L, Millot GA, Charpin A, Laugé A, Tarabeux J, et al. Mosaicism and prenatal diagnosis options: insights from retinoblastoma. Eur J Hum Genet. 2017;25:381-3. 